\title{
Australia y el Pacífico
}

\begin{abstract}
B R U CE GRAN'T, escritor y periodista australiano, fue profesor de Ciencias Políticas en la Universidad de Melbourne. Ha publicado numerosas obras de ficción y políticas. Sus artículos han aparecido en diversas revistas como Foreign Affairs y Pacific Community. Entre sus obras más importantes se cuenta el libro Indonesia, publicado por la Universidad de Melbourne y posteriormente por Penguin, que ganó el principal premio australiano de literatura en 1964. El señor GRANT es colaborador regular del diario Washington Post y está próximo a publicar un nuevo libro sobre política exterior australiana.
\end{abstract}

Algunos escritores japoneses han comenzado a utilizar la expresión "defensa defensiva", presumiblemente para diferenciarla de lo que en ese país ha sido descrito comúnmente como "defensa ofensiva", refiriéndose a sus fuerzas militares y su posible papel regional. Los australianos están entregados a un debate comparable, que se expresa en la controversia entre aquellos que mantienen las ventajas de una "defensa adelantada" y quienes sostienen la tesis de una "defensa continental" o "fortificada" (fortress Australia). El significado de este debate y sus relaciones con las actitudes de los australianos hacia el ámbito exterior podrán ser juzgados al examinar sus implicancias frente a la posición tradicional de esta nación frente a los problemas de su seguridad militar y política.

Australia ha llegado a la edad adulta como nación doscientos años después de la primera ocupación territorial británica; más de un siglo desde que las colonias originales se convirtieran en estados separados y autónomos, y setenta años después de que la nación fuera fundada bajo un régimen de protección benigna por parte de la potencia metropolitana. De aquí que aun cuando la lucha por la supervivencia física ha sido evidentemente ardua en Australia, nunca ha habido una lucha política similar contra Gran Bretaña. Las motivaciones antibritánicas que se encuentran sin dificultad en el cauce cultural nacionalista australiano, aunque persistentes, nunca han logrado adquirir validez política. En verdad, los australianos son harto diferentes de los británicos en sus modales y estilo, pero han conservado hasta época muy reciente un gran afecto y admiración por las cosas británicas. 
Esto estaba basado en una emoción muy simple: al temor de que si ellos, los australianos, no se hacían de alguna manera indispensables a los británicos, podrían ser abandonados a su propia suerte en una parte distante y hostil del mundo.

Por lo mismo, Australia apoyó abiertamente a Gran Bretaña en sus conflictos en todo el mundo durante la mejor parte de un siglo: en el Sudán, en la guerra de los Boers, en las dos guerras mundiales, en Malaya y clespués en Malasia. Detrás del mismo objetivo, leal a su protector, Australia ha intervenido también junto a los Estados Unidos en Corea y en Vietnam.

Esta política de apoyo al brazo y al espíritu del protector no era una política insincera ni desatinada; era realista. En verdad los británicos protegieron a los australianos hasta la Segunda Guerra Mundial y los ayudaron a obtener uno de los más altos niveles de vida material del mundo. En el mismo sentido fue realista buscar la ayuda de Estados Unidos contra los japoneses en la Segunda Guerra Mundial y su protección generalizada en el inestable período de posguerra, cuando las potencias europeas se estaban retirando del Sudeste Asiático. Los británicos no eran más lo suficientemente poderosos; las colonias europeas en Asia no sobrevivirían; el nacionalismo asiático era un factor desconocido; en cambio, los Estados Unidos, la nación más poderosa del mundo y un aliado en tiempo de guerra, estaba a mano.

Además -y lo más importante para los australianos- los Estados Unidos tenían lo que el Primer Ministro Gorton llamó "el mismo enfoque respecto a la vida" de nosotros los australianos. Esta similitud mental con el protector tiene una atracción casi mística para muchos australianos. Incluye, además de la democracia, todas esas cosas que no son mencionadas habitualmente en los discursos internacionales: raza, capitalismo y cristianismo. Esto significaría para los australianos que la influencia del protector, que no puecle ser evitada, es algo benigno.

Probablemente, el factor que más pesa en las actitudes tradicionales australianas hacia el Asia, es la raza. Es imposible - por lo menos es imposible para un australiano- imaginar a los australianos aceptando protección de una nación poderosa que no tuviera el "mismo enfoque respecto a la vidla". El único candiclato posible, si los Estados Unidos decayeran, es la Unión Soviética. El año 1969, durante la campaña electoral parlamentaria, el Gobierno publicó una declaración en la que sugería de una manera muy indirecta que la Unión Soviética podría quizás ofrecer algo constructivo para la estabilidad del Sudeste Asiático. La reacción de la oposición-de derecha y de izquierda- fue notable. Se dijo que las políticas extranjera $y$ de defensa del gobierno estaban en tal confusión, a 
causa de los reveses de los estadouniclenses en Vietnam y la debilitada presencia de Gran Bretaña al "este de Suez", que se estaba inclinando a pedir protección a la Unión Soviética. El Gobierno tuvo que desdecirse, declarando su imperecedera oposición al comunismo $y$ sus reticencias de los motivos que guían a la cliplomacia soviética. Mucho de lo que se convirtió en un incidente pasajero y jocoso, se debió sin duda a la atmósfera de lá campaña electoral, pero aclaró la común creencia australiana de que el país debe tener una nación protectora.

Esta iclea de un protector para la clefensa y para la política exterior de Australia, explica la clefensa inadecuada y la combinación resultante de complacencia y agitado senticlo de peligro y crisis; ayucla a explicar también por qué el debate sobre la "clefensa adelantada" se ha converticlo en la piedra de toque del pensamiento australiano sobre sus responsabilidades y oportunidades en Asia y en el Pacífico en la década cle 1970. Porque la "defensá adelantada" era un perfeccionamiento y una consolidación en el Sudeste Asiático de la política expedicionaria que Australia había llevado hasta la Seguncla Guerra Mundial. Incapaz de defenderse por sí misma contra el tipo de agresión que habían lanzado los japoneses, el núcleo central de la política de Australia fue mantener a los británicos y a los norteamericanos comprometidos en el Sudeste Asiático, con fuerzas interpuestas entre el continente australiano y cualquier "amenaza clel Norte". El perímetro de la defensa de Australia, en Malasia y Singapur (con los británicos) y en Thailandia, y Vietnam del Sur (con los estadounidenses) sólo podía ser manteniclo con la ayuda de aquellos que llegaron a ser conocidos, en la refulgente frase del Primer Ministro Menzies, como "nuestros grandes y poderosos amigos".

Cuanclo en 1968 el gobierno laborista británico deciclió retirar sus fuerzas de Malasia-Singapur después de 1971 y los norteamericanos invirtieron su política en Vietnam, el concepto de "defensa adelantada" se hizo añicos. Los australianos reconocieron que no podían mantener ese perímetro defensivo con sus fuerzas propias, que no estaban preparados para proponer una especie de "doctrina Monroe" para el Sudeste Asiático y que la idea de estar involucraclos en los asuntos del Sudeste Asiático, que había sido un artículo de fe clurante veinte años, necesitaba ser reexaminada. Este examen está aún realizándose. Uno de los azares que hay que correr al escribir sobre la defensa y la política extranjera de Australia en este momento, si uno se cla cuenta que Australia, como porler ascendente en el Sudeste Asiático, tiene derecho a decir más de algo en lo que ocurre en la región en cuanto a su seguridad, es que no 
hay certeza en este momento cómo Australia podrá ejercer ese derecho.

El debate de 1968 llegó a su cúspide en febrero de 1969, cuando el gobiemo anunció su intención de mantener fuerzas australianas en Malasia-Singapur después que los británicos se retiraran en 197l. Fue una decisión histórica, porque significaba que Australia intentaba mantener fuerzas en el Sudeste Asiático sin la protección de amigos grandes y poderosos. Después de la decisión, sin embargo, la resolución fue sacudida por motines raciales en Malasia, en mayo de 1969, y por el anuncio del Presidente Nixon, al mes siguiente, sobre los principios de la política norteamericana en Asia que han sido llamados la "Doctrina de Guam". Los motines raciales hicieron temer a Australia que sus tropas podrían verse envueltas en una guerra civil ( $y$ comunal) en Malasia. La Doctrina de Guam, al dar importancia a la necesidad de "autoconfianza" y particularmente al establecer la renuncia norteamericana a comprometer sus fuerzas de combate contra la insurgencia, revivió en los australianos el temor de que ellos se verían en apuros en Malasia-Singapur, clonde estarían comprometiclos a luchar contra la insurgencia.

In las elecciones parlamentarias de octubre de 1969, sobre la base cle un programa cle retiro unilateral cle Vietnam, el no envío de fuerzas terrestres a Malasia-Singapur (y sólo una insinuación de promesa de ayucla aérea y naval), y la abolición de la conscripción militar, el Partido Laborista obtuyo un inesperado buen resultado, redujo la mayoria del gobierno, en un Parlamento de 125 representantes, de 38 a 7 asientos y produjo una revitalización clel sentimiento liberal izquierdista que podría incluso llevar al poder a los laboristas en las próximas elecciones de 1972.

En estas circunstancias, parece que ningún particlo tratará de capitalizar una política de participación en los asuntos del Sudeste Asiático, cosa que ahora aparece como una política agotada. Los dos partidos mayoritarios están clivididos respecto a las políticas exterior y de defensa. Las filas del gobierno conservador incluyen a algunos que favorecen el repliegue de las fuerzas australianas desde el Sucleste Asiático hacia un continente "fortaleza" protegido por una fuerza nuclear australiana. La oposición laborista, que ha estaclo fuera del poder desde 1949 y tiende a ser ideológica respecto cle la política exterior y de defensa también ha adoptado la idea de la clefensa "continental", pero algunos de sus líderes más pragmáticos favorecen una especie de sistema de defensa "regional", al cual Australia podría contribuir junto con Indonesia, Malasia y Singapur, con equipo, asesoría técnica, intercambio de información, entre- 
namiento y maniobras conjuntas, pero no con el envio al extranjero de fuerzas australianas.

Antes era posible caracterizar las posiciones políticas australianas usando como elementos comprobatorios la "política de defensa adelantada" (PDA) y a la "política de la Australia Blanca" (PBA). Si usted era partidario tanto de la PDA como de la PBA usted era conservador, anticomunista y nacionalista. Si lo era de la PDA y estaba en contra de la PBA, quería decir que usted era liberal, anticomunista e internacionalista. Estar en contra de la PDA y en favor de la PBA hacía de usted un izquierdista, nacionalista y aislacionista. Si estaba en contra de la PDA y de la PBA era usted un liberal, internacionalista y, probablemente, un pacifista. Mientras el segundo grupo tuvo la ventaja sobre el primero en la sucesión de gobiernos conservadores después de $1949 \mathrm{y}$, por tanto, la intervención en Asia condujo a la correspondiente liberalización de la restricción en la inmigración, el tercer grupo tiene la ventaja sobre el cuarto en la política laborista $y$, por tanto, si hay un cambio de gobierno en 1972 y un retiro de las fuerzas militares australianas de Asia, no habría una ulterior liberalización en la inmigración. En una palabra, si la inmigración es tomada como un test crítico de la capacidad de Australia para ponerse de acuerdo con su geografía, un gobierno laborista será incapaz de hacer historia, por lo menos en este sentido.

Al mismo tiempo que las políticas externas de Australia fueron sometidas a un examen crítico, el público australiano fue adquiriendo la noción de que la "calidad de la vida" en Australia se estaba deteriorando. A corto plazo - por lo menos en lo que concierne a beneficios como servicios sociales, educación, conservación y lucha contra la contaminación- la atención del público debe pasar de la percepción de amenazas externas y la necesidad de políticas de seguridad a la percepción de desigualdades internas, injusticia e incompetencia y a la necesidad de una legislación interna imaginativa y costosa como un correctivo.

Los australianos se preocupan ahora más de la desigualdad entre sus propios ricos y pobres que de la brecha entre naciones ricas y pobres. Se preocupan más de la represión en su propio país que de la represión en los países comunistas. Is más fácil atraer la atención de las masas con planes para conservar los recursos naturales y detener la contaminación arnbiental que con planes para una defensa más poderosa o para iniciativas en política exterior. Sin duda, esta vuelta a sus problemas internos podría detenerse dramáticamente si hubiera una nueva y notoria amenaza a Australia. Pero mientras más australianos vuelven las espaldas a las complejidades de Asia, más fácil es no darse cuenta de posibles amenazas. Hace 
diez años, la caída de una aldea de Laos en manos de las fuerzas comunistas aparecía a las masas australianas como una parte de un proceso que, si no era detenido, podría alcanzar hasta sus mismas playas. Hoy día, las masas se han hecho más críticas y escépticas sobre la penetración "dlescendente" del comunismo en el Sudeste Asiático; la teoría clel clominó y la necesidad de contener a la Ghina comunista $\mathrm{y}$ otras consideraciones estratégicas de la guerra fría. Flay mayor interés entre la mayoría de los australianos y ciertamente en los círculos comerciales, por la penetración económica en el Sucleste Asiático y en Australia de una nación amiga, Japón, que en los potenciales subversivos, o agresivos, por parte de cualquiera de los presuntos adversarios como China y la Unión Soviética.

En ausencia de otro conflicto como el de Vietnam, que hizo de la seguridad exterior la cuestión palpitante en la elección de 1966, u otra confrontación clel tipo de la de Indonesia, que provocó la amenaza de guerra en la elección de 1963, la próxima elección en Australia va a ser, presumiblemente, referida fundamentalmente a cuestiones domésticas. Una de estas cuestiones puede ser la de la mantención de la ley y el orclen internos, en relación al movimiento de protesta que ha surgiclo en oposición a la guerra de Vietnam. Esto podría ayudar al gobiemo, pero sin aliados listos para actuar concertadamente en el Sucleste Asiático, el triunfo de un gobierno conservador en Australia en 1972 no volverá a dar a la política exterior y de clefensa un papel principal en la política australiana, como ocurrió clurante la guerra fría. En realidad, al tener que apoyarse en el problemático futuro de una política exterior "adelantada", Ios conservadores pueden verse obligados a ser más restrictivos en Ias iniciativas de su política exterior que el Particlo Laborista, que tiene una traclición universalista y humanitaria.

La victoria de los "tories" en las elecciones británicas de 1970 ha influido en la confianza con que Australia se proyecta en el Sudeste Asiático. Los riesgos para Australia no son tan grandes si los británicos, independientemente cle la canticlacl de sus fuerzas están dispuestos a mantener una "presencia militar" en Malasia-Singapur. Pero esta influencia es más psicológica y temporal que política y permanente. Los británicos, los australianos y los neozelandeses no están concertando un compromiso que signifique mantener una fuerza decisiva en el desarrollo clel equilibrio del pocler en Asia. Este equilibrio será finalmente establecido por los Estados Unidos, la Unión Soviética, China y Japón aun cuando, tarcle o temprano, requerirá una respuesta de Australia y sus aliados. Una presencia reducida de Gran Bretaña en Malasia-Singapur sería importante para dar a Australia - $y$ a Malasia-Singapur- tiempo para preparar 
una política más decidicla. Podría dar también a los Estados Unidos tiempo y confianza para desentenderse del Sudeste Asiático sin poner de relieve un viraje hacia un aislacionismo total. Pero los cambios que ya están ocumiendo en esa región son sustanciales y el esfuerzo que Gran Bretaña puede hacer, aun si el Gobiemo de Mr. Heath puede realizar lo que se ha dicho que hará en SingapurMalasia, difícilmente harán una impresión duradera en ellos. Esto será especialmente el caso ahora que Gran Bretaña se ha incorporado al Mercado Común Europeo.

Para resumir: la ecuación lealtad-protección ha cesaclo de tener vigencia para Australia como factor para su política de seguridad en el Sudeste Asiático. No hay ningún sustituto preparado. Se puede ver que Australia ha siclo arrastrada a una "defensa adelantada"; a la preocupación por la estabilidad y seguridad del Sudeste Asiático, por una necesidad profundamente senticla de protección de un contorno extraño que se presume hostil. La única manera que esta posición adelantada puede ser preservada por Australia sola es, presentándola públicamente, no como una respuesta a una amenaza, sino como una oportunidad para jugar un papel nacional en un ámbito regional, y esto, antes de que la apreciación de la opinión pública australiana valore la capacidad nacional para jugar tal papel.

La clependencia es una estrategia conservaclora. No es, por lo tanto, sorprendente que gobiernos conservadores hayan estado en el poder en Australia clesde 1949, y que a ninguna directiva de estos gobiernos se le haya ocurriclo que Australia podría tratar de poseer una voz independiente en los asuntos internacionales. Durante los últimos veinte años, la finalidad de la política exterior ha sido buscar aliados poderosos y el interés particular de Australia ha estado subordinado a los intereses generales de esos aliados. Para realizar esto con alguna consistencia, ha sido necesario para los gobiernos australianos aunar a un alto nivel de generalización los intereses comunes de Australia $y$ sus protectores. Al clejar establecido que tenemos tanto en común, no había necesidad ni lugar en Australia para criterios indepenclientes. El criterio sustancial se establecía en Londres y Washington. Algunas veces podíamos contribuir con argucias, correcciones o sugerencias, pero privadamente, no en público. La política exterior australiana ha llegado a ser así fundamentalmente una cuestión de consultas; el más importante objetivo de muestra diplomacia, el que la consulta se efectúa antes y no después de que se adopten las decisiones. Estábamos en los corredores del poder, pero no para plantear cuáles eran los intereses australianos, sino para escuchar de nuestros poderosos aliados cuáles eran los intereses comunes de la alianza. 
Se han tomado iniciativas periféricas. Gracias a una diestra diplomacia personal, Australia mantuvo estrechos contactos con Camboya y Sihanouk a pesar de nuestras igualmente estrechas relaciones con Saigón y Washington. A pesar de poderosas presiones y la irritación popular en Australia con la política del gobierno de Sukarno, Camberra logró conservar buenas relaciones con Jakarta durante la confrontación con Malasia, aun cuando fuerzas australianas esturieran en ese tiempo en las fronteras de Malasia preparadas para resistir a los indonesios. Australia ha logrado también una "relación especial" con Malasia y Singapur.

No quiero sugerir que tales logros sean despreciables, pero creo que se han conseguido a pesar de las tendencias de la política australiana. Sea como fuere, algunas personas inteligentes y perspicaces del Departamento de Asuntos Exteriores, o críticos en universiclades, periódicos $u$ otras instituciones, lograron promover con esfuerzo, al mismo tiempo, una actitud australiana independiente hacia el Sudeste Asiático a pesar de, y no a causa de, la política dominante.

Quizás también por falta de una dirección política, el concepto de una política exterior nacionalista es algo que un australiano no comprende de inmediato. En primer lugar, la idea de nación no es fuerte. Los australianos tienen un fuerte sentido de arraigo a su tierra y un creciente sentido de comunidad humana, pero no un sentido de nación. De hecho, para muchos australianos no está claro cuándo comenzó la nación. Algunos piensan que fue el día que los británicos fundaron el establecimiento penal en Australia; otros piensan que fue cuando se formó el Commonwealth a partir de los estados coloniales anteriores; otros más que fue aquel día en una playa en Gallipoli durante la Primera Guerra Mundial, cuando Australia experimentó su primer encuentro armado mayor que, aunque un fracaso, fue un fracaso glorioso considerado como el origen del sentido de nacionalidad australiana. Hay todavía otros que creen que Australia peleó para nacer como nación en Ballarat, en 1854, cuando los mineros del oro se rebelaron contra la autoridad y levantaron un bandera, poniendo la Cruz del Sur contra un fondo de azul cielo, durante el incidente de Eureka Stockade. Pero la mayoría de los australianos parecen indiferentes a esta búsqueda de un alma nacional. A pesar del crecimiento de Australia y su evidente capacidacl como una nación que interviene en los asuntos internacionales, tienden a ser pasivos considerando que Australia es más un objeto que un sujeto de la política internacional.

Además, el concepto de un papel activo no es familiar a los australianos. Este requiere una acción coherente, una posición pre- 
clecible e identificable respecto de los problemas del mundo. Requiere también que quienes atribuyan ese papel sean otras naciones. Los australianos tienen dudas de cómo los ven otros pueblos, especialmente sus vecinos. Los australianos no tienen ese deseo irracional de construir una gran nación, que es el que empuja a un pueblo hasta los límites de la naturaleza humana. Sus aspiraciones son generalmente pragmáticas, no son apasionadas excepto respecto: a la supervivencia. Tampoco tienen la competencia y experiencia político-diplomática que permitiría realizar aquellas iniciativas útiles aun cuando modestas que pueden hacer de una nación pequeña un poder significativo en el ámbito de las relaciones internacionales.

A esta lista de aspectos aparentemente deplorables hay que agregar sin embargo, que un creciente número de intelectuales están entusiasmados con la perspectiva de una Australia que escapa del paraguas del protector y participa en un sofisticado juego de intereses nacionales entre los poderes rivales de Asia, especialmente del Sudeste Asiático. Uno de los argumentos más convincentes para la existencia de fuerzas en Malasia-Singapur es que podrían habilitar a Australia, con una presencia militar simbólica, para ejercer una influencia política en la región.

Esta es quizás la forma más sofisticada de nacionalismo que se haya clesarrollado hasta aquí en Australia y no constituye de ningún modo una reversión a valores provinciales y rurales. Sus partidarios son el producto de la opulencia; creen más en una cultura mundial que en un nacionalismo australiano o en una identidad regional. Pero ellos están haciendo de la participación australiana en los asuntos del Sudeste Asiático, la llave para la reaparición de un sentido de dignidad y confianza en la nación australiana ya que se alega que sólo tomando para sí la tarea de la responsabilidad nacional en el Sudeste Asiático, puede Australia desarrollarse como una nación. Quizás esto tenga algo de romántico. No es fácil, con la sombra del Vietnam, hacer que la mayoría de los australianos -y esto incluye a los partidos políticos- vean en el desafío que hay para Australia en el Sudeste Asiático algo positivo en vez de algo sombrio y aterrorizador. La duda y la incertidumbre que he descrito pueden revelar a un extraño los síntomas de una nación en crisis y esto puede ser verdad. Me doy cuenta, sin embargo, que Australia nunca va a permitir ser inmovilizada por alternativas apocalípticas. Una crisis es también una encrucijada. A causa del desarrollo económico de Australia y de sus intereses globales como una nación comerciante de importancia acrecentados por la distensión de la camisa de fuerza de la política de la Guerra Fría, parece improbable que la nación pueda resistir indefinidamente la invitación a definir sus 
intereses y à movilizar el apoyo popular para la política exterior $y$ cle clefensa que promueven esos intereses.

Asumiendo que el examen introspectivo de los australianos tendrá que llevar a una definición y que a esta seguiría eventualmente una retractación de algunos compromisos vigentes, ¿dónde buscarán los australianos la oportunidad para expresar un creciente sentido de independencia? Las tres zonas que desafían a la política australiana son el Océano Indico, el Sudeste Asiático y la zona del Pacífico.

Últimamente el Océano Indico ha provocado una gran atención descle un punto de vista político en Australia a causa de la disminución del poderío naval británico, de la presión de la Unión Soviética en el Mediterráneo y el Medio Oriente tanto como cle su influencia en el subcontinente índico y su creciente interés naval en el Océano Indico mismo. El problema de todos los esquemas propuestos para un papel de Australia en el Océano Indico es que requieren una especie de ligazón con Sudáfrica. Hay una corriente de opinión en Australia con influencia en los particlos de gobierno, a la cual le gustaría que se estableciera un acuerclo defensivo entre Australia y Sudáfrica. La clesventaja enorme de un arreglo de este tipo es que complicaría las relaciones de Australia con las naciones del Sudeste Asiático. Mi impresión es que la mayoría cle los australianos se sentirían muy incómodos con una relación especial con Sucláfrica, no sólo a causa de su política racial, sino porque los australianos están clesarrollando un tipo cliferente de sociedad; más abiertamente materialista y tolerante que el rígiclo y paternalista modo de vida que el "establishment" sudafricano parece considerar como el nervio de su civilización. El sentimiento general, particularmente vivo entre los jóvenes, de que Sucláfrica es un país de moralistas anacrónicos, combinado con una estimación técnica de que una relación estrecha con Sudáfrica nos haría sospechosos en e] Sudeste Asiático, probablemente basta para eliminar, como una posibilidad seria, una alianza entre Sudáfrica y Australia, a través del Océano Indico. Mientras Australia pueda muy bien, como medida de autodefensa, ocuparse de la protección de sus costas occidentales, no tiene la capacidad para desarrollarse como un poder en el Océano indico excepto como parte de un sistema regional. La insistente candidatura de Sudáfrica de ser un aliado, ofrece un precio demasiado alto para que Australia llegue a ser un miembro de tal sistema.

Se pueden avanzar muchas razones del por qué Australia podría tomar una parte activa en los asuntos del Sudeste Asiático. Tenemos estrechas relaciones con países de esa zona, hemos desarrollado intereses especiales en esa región, y hemos terminaclo por reconocer 
que es una parte del mundo en la cual la seguridad de Australia puede verse afectada críticamente.

Pero hay dos problemas en el papel de Australia en el Sudeste Asiático. Uno es que es una región de la cual Australia puede esperar poco. Sus países necesitan asistencia en varias formas: desarrollo, capital, apoyo militar y ayuda técnica. Cuando los australianos miran hacia el Sucleste Asiático, ven una parte del mundo que algo quiere de Australia como un dador y un proveedor. Frente a los asuntos y a las demandas internas mencionadas antes, es discutible si las actitudes generosas hacia el Sudeste Asiático pueden ser mantenidas si disminuyen las amenazas dentro y hacia la región.

El segundo problema es que los pueblos del Sudeste Asiático pueden desarrollar formas regionales y comunitarias de las cuales Australia estaría excluida. Es interesante, por ejemplo, que Australia es un miembro de la ASPAG, un grupo formado en Seul por iniciativa del Japón, mientras que no es miembro de la ASEAN, que está mucho más cerca de ella. La razón para no ser miembro de la ASEAN, me imagino, es que Indonesia, en particular, mira a esta asociación como un foco local donde su gravitación es principalísima. La ASEAN tiene una personalidad cultural que podría diluirse si Australia se incorporara.

Puede haber buenas razones para impulsar a Indonesia a sentirse segura y activa en un papel más limitado que aquel proyectado por el Presidente Sukarno, quien siempre vio a su país entre las grandes naciones. Desde el punto de vista de Australia, puede ser más valioso cultivar relaciones bilaterales que tratar de forzar el paso de su aceptación en el Sudeste Asiático como un país de origen europeo y una marcada diferencia en el nivel de vida. Es valioso permanecer al borde del regionalismo, dando apoyo y asistencia, quizás estancándose en el proceso, pero sin tratar de perder una identidad perfectamente distinta en consideración de una aceptación cultural momentánea, que puede ser insincera en ambos lados y que puede tenei un efecto político desestabilizador.

El Pacífico, en contraste, tainto con el Océano f́ndico como con el Sudeste Asiático, es para Australia, fuente de inspiración y poder.

Estados Unidos es indiscutiblemente un poder en el Pacífico. Aunque sus fuerzas se reducen en Europa y en el Sudeste Asiático y por muy reticente que sea a contraer compromisos adicionales en el Océano fndico, su presencia dominante en el Pacífico parece asegurada. El documento más importante en la seguridad de Australia es el tratado ANZUS, que incluye a los Estados Unidos, Nueva Zelandia y Australia en un acuerdo de defensa mutua (aunque unilateral). Las cláusulas del ANZUS se refieren no sólo a ataques a territorios metropolitanos $\mathrm{y}$ posesiones de los signatarios, sino 
también a sus "fuerzas armadas, navales o fuerza aérea", en lo que es llamado, aunque no definido, "zona del Pacífico". Mientras que el tratado de la OTASO que concierne al Sudeste Asiático, es resistido en Australia, el de ANZUS, la base de cualquier protección estadounidense a Australia, tiene el apoyo cle ambos partidos y es ampliamente aceptado por la población. Además las relaciones con los Estados Uninidos son más que una alianza militar. Así como en el pasado el Océano Indico y el Canal de Suez proveyeron a Australia de una línea de conexión vital con el Imperio Británico, la seguridad del Pacífico es hoy día esencial para los importantes lazos comerciales y culturales de Australia con los Estados Unidos.

Japón ha llegado a ser el primer cliente comercial de Australia. La dependencia de Australia del progreso económico japonés es uno de los hechos concretos de la situación contemporánea de los cuales los políticos australianos recién empiezan a darse cuenta. Tome la forma política que tome esta relación - si el Japón y Australia se combinan, por ejemplo, en el Sudeste Asiático, para desarrollar la región- el comercio entre ambos países es un comercio del Pacífico. Japón tiene un punto de vista complejo sobre Asia; tiene un claro interés en el stalu quo en Corea y Taiwan, pero en oposición a esto, tiene una relación casi simbólica con China y es cortejado por la Unión Soviética. Parece preocuparse poco por el desarrollo del subcontinente inclio; tiene desde hace tiempo interés en las materias primas y mercados del Sucleste Asiático y recientemente ha agregado a esto un compromiso de ayuda a la región a través del Banco de Desarrollo Asiático. Pero es aún resistido políticamente en el Sudeste Asiático. Debido a su profunda necesidad del mercado norteamericano, Japón va a permanecer fundamentalmente, en la clécada próxima, como una nación del Pacífico.

La especial responsabilidad de Australia por Nueva Guinea, nos inclinará hacia el Pacífico. Es realista pensar que Australia estará ligada al destino de Nueva Guinea después de su independencia. Lógrese o no un arreglo defensivo, Australia estará interesada en las relaciones que Nueva Guinea establezca con las naciones que la rodean. Más importantes serán las relaciones con Indonesia. Pèro una perspectiva que yo pienso que Australia debe impulsar, es el establecimiento después de Ia independencia de Nueva Guinea, de lazos de seguridad entre esta última con las islas del área del $\mathrm{Pa}-$ cífico, en el presente en varios grados de desarrollo. Como dijimos antes, el Pacífico es la zona declarada del tratado del ANZUS y tanto los Estados Unidos como Nueva Zelandia tienen responsabilidades en los territorios insulares del Pacífico.

La estrecha relación de Australia con Nueva Zelandia nos em- 
puja hacia el Pacífico. No hay un empuje semejante desde el Océano Indico. Relaciones de seguridacl entre Australia y Nueva Zelandia, no sólo en una forma bilateral sino en la forma de una asociación en el Sucleste Asiático, han sido tan estrechas como pueden serlo para dos estaclos soberanos.

Para volver al tema de este trabajo, el Pacífico ofrece a Australia algún alivio a los problemas de la posición adelantada en el Sudeste Asiático; presenta al protector disfrazado de miembro dirigente de una comunidad indefinida; ofrece riqueza, poder amistoso y una variedad cultural $y$ racial, que es atractiva a los australianos que se sienten bajo la presión de Asia y Africa. No ofrece organización regional, pero no estoy seguro si la inclinación australiana no podría ser la valorización positiva de esta omisión, ciertamente cuando el sentimiento del país está en contra de nuevos compromisos externos. Me refiero a una organización de seguridad, no a una organización económica.

Este trabajo pretencle proveer de una introducción a la conducta exterior de Australia, la cual no es normalmente un tema de cliscusión internacional. Me parece que el resultado contiene un núcleo de verdad. Esto es que los australianos están reconsiderando su seguridad y emprendiendo un cambio en sus actitudes hacia sí mismos y hacia el mundo no menos profundo que el que afronta a los Estados Unidos. Una potencia mediana tiene límites estrictos en su libertad para actuar en el ámbito externo, pero su libertad para retirarse está limitada solamente por sus propias percepciones. Es, sin embargo, difícil estar seguro cuál será la futura política australiana. La instrumentalización de una política cle compromisos puede ser rápidamente desmantelada, pero la continuidad oficial permanece mucho después de que este cambio ha sido aceptado $y$ apoyado por la ciudadanía.

La conclusión preliminar de este artículo es que, de las tres áreas abiertas a la política activa de Australia -Océano Índico, Sudeste Asiático, Área del Pacífico- la última ofrece las mejores oportunidades y posiblemente la respuesta más acertada.

Aunque, según mi punto de vista, existe un conflicto entre los papeles de Australia en el Océano Indico y en el Sucleste Asiático, tal conflicto no existe para Australia entre el Pacífico y el Sudeste Asiático. Las conexiones de Australia con el Pacífico le darían fuerza para comprometerse de nuevo en Asia, especialmente en el Sudeste Asiático. Si la conexión con el Pacífico da a los australianos una certeza de seguridad, la necesidad de medidas militares en el Sudeste Asiático, que constituye un dilema inevitable para el aislacionismo australiano, puede ser examinada bajo condiciones de menor tensión y urgencia. 